\title{
Effective Dynamic Detection of Alias Analysis Errors
}

\author{
Draft - The camera-ready version will be ready soon. \\ Jingyue Wu, Gang Hu, Yang Tang, Junfeng Yang \\ Columbia University \\ \{jingyue,ganghu,ty,junfeng\}@cs.columbia.edu
}

\begin{abstract}
Alias analysis is perhaps one of the most crucial and widely used analyses, and has attracted tremendous research efforts over the years. Yet, advanced alias analyses are extremely difficult to get right, and the bugs in these analyses are one key reason that they have not been adopted to production compilers. This paper presents NEONGOBY, a system for effectively detecting errors in alias analysis implementations, improving their correctness and hopefully widening their adoption. NEONGOBY works by dynamically observing pointer addresses during the execution of a test program and then checking these addresses against an alias analysis for errors. It is explicitly designed to (1) be agnostic to the alias analysis it checks for maximum applicability and ease of use and (2) detect alias analysis errors that manifest on real-world programs and workloads. It emits no false positives as long as test programs do not have undefined behavior per ANSI C specification or call external functions that interfere with our detection algorithm. It reduces performance overhead using a practical selection of techniques. Evaluation on three popular alias analyses and real-world programs Apache and MySQL shows that NEONGOBY effectively finds 29 alias analysis bugs with zero false positives and reasonable overhead; four of the bugs have been patched by the developers. To enable alias analysis builders to start using NEONGOBY today, we have released it open-source at https://github.com/ wujingyue/neongoby, along with our error detection results and proposed patches.
\end{abstract}

\section{Categories and Subject Descriptors}

D.2.4 [Software Engineering]: Software/Program Verification; D.2.5 [Software Engineering]: Testing and Debugging; F.3.2 [Logics and Meanings of Programs]: Semantics of Programming Languages

\section{General Terms}

Algorithms, Design, Reliability, Experimentation

\section{Keywords}

Error Detection, Alias Analysis, Dynamic Analysis

\section{INTRODUCTION}

Alias analysis answers queries such as "whether pointers $p$ and $q$ may point to the same object." It is perhaps one of the most crucial and widely used analyses, and the foundation for many advanced tools such as compiler optimizers [27], bounds checkers [18], and verifiers $[14,13]$. Unsurprisingly, a plethora of research [30, 22, 23 ] over the last several decades has been devoted to improve the precision and speed of alias analysis, and PLDI and POPL alone have accepted over 37 alias analysis papers since 1998 [29]. (Most citation lists in this paragraph are seriously incomplete for space.)

Unfortunately, despite our reliance on alias analysis and the tremendous efforts to improve it, today's production compilers still use the most rudimentary and imprecise alias analyses. For instance, the default alias analysis in LLVM for code generation, basicaa, simply collapses all address-taken variables into one abstract location; the default alias analysis in GCC is type-based and marks all variables of compatible types aliases. These imprecise analyses may cause compilers to generate inefficient code.

We believe one key reason hindering the adoption of advanced alias analyses is that they are extremely difficult to get right. Advanced alias analyses tend to require complex implementations to provide features such as flow sensitivity, context sensitivity, and field sensitivity and to handle corner cases such as $\mathrm{C}$ unions, external functions, function pointers, and wild void* and int casts. As usual, complexity leads to bugs. Buggy alias results at the very least cause research prototypes to yield misleading evaluation numbers. For instance, our evaluation shows that LLVM's anders-aa, implementing an interprocedural Andersens's algorithm, is actually less precise than basicaa (\$8.1.1) after we fixed 13 anders-aa bugs. Worse, buggy alias results cause optimizers to generate incorrect code, commonly believed to be among the worst possible bugs to diagnose. Moreover, they compromise the safety of bounds checkers and verifiers, yet this safety is crucial because these tools often have high compilation, runtime, or manual overhead, and are applied only when safety is paramount.

This paper presents NEONGOBY, ${ }^{1}$ a system for effectively detecting errors in alias analysis implementations, improving their correctness and hopefully vastly widening their adoption. We explicitly designed NEONGOBY to be agnostic to the alias analysis it checks: the only requirement is a standard MayAlias $(p, q)$ interface that returns true if $\mathrm{p}$ and $\mathrm{q}$ may alias and false otherwise. ${ }^{2}$ This minimum requirement ensures maximum applicability and ease of use. To check an alias analysis with NEONGOBY, a user additionally chooses a test program and workload at her will. For instance, she can choose a large program such as Apache and MySQL and a stressful workload that together exercise many diverse program constructs, such as the corner cases listed in the previous paragraph. This flexibility enables NEONGOBY to catch alias analysis bugs that manifest on real-world programs and workloads.

Given the test program, NEONGOBY instruments the program's pointer definitions to track pointer addresses. To increase check-

\footnotetext{
${ }^{1}$ We name our system after the neon goby fish which helps other fish by cleaning external parasites off them.

${ }^{2}$ NeONGoby can be easily extended to check must-alias but few alias analyses implement a more-than-shallow must-alias analysis.
} 


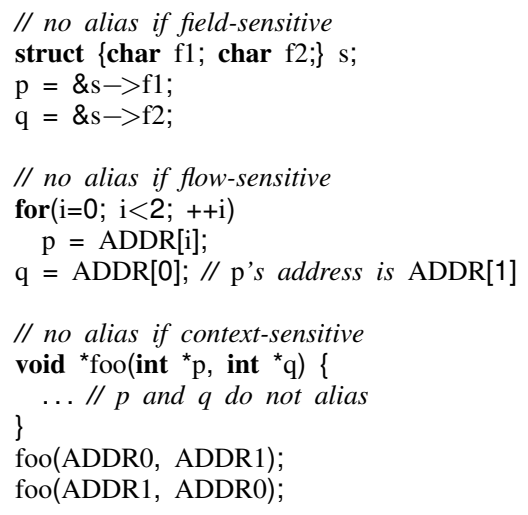

Figure 1: False positive examples caused by sensitivities.

ing coverage, NEONGOBY instruments all pointer definitions including intermediate results, e.g., pointer arithmetics. The user then runs the instrumented program on the workload, and NEONGOBY dynamically observes pointer addresses (henceforth referred to as addresses) and checks them against the alias analysis. It emits bug reports if the addresses contradict the alias results, i.e., the pointers did alias during the test run but the alias analysis states that the two pointers never alias. We use $\operatorname{DidAlias}(p, q)$ to refer to NEONGOBY's algorithm for determining whether pointers $p$ and $q$ did alias. The invariant NEONGOBY checks is thus $\operatorname{DidAlias}(p, q) \rightarrow \operatorname{MayAlias}(p, q)$. To ease discussion, we use DidAlias/MayAlias to refer to both the corresponding algorithm and the set of pointer pairs on which DidAlias/MayAlias returns true.

Although the idea of dynamically checking alias analysis enjoys conceptual simplicity, implementing it faces a key challenge: how to reduce false positives, a major factor limiting the usefulness and adoption of error detection tools [10]. False positives arise from two main sources:

First, a naïve DidAlias algorithm may be less precise than the alias analysis checked. Figure 1 shows three examples on which an imprecise DidAlias may emit false positives. Specifically, if an imprecise DidAlias considers pointers with one byte apart as aliases (because they likely point to the same object), it may emit a false positive for a field-sensitive alias analysis on the first example; if it considers pointers ever assigned the same address as aliases, it may emit a false positive for a flow-sensitive alias analysis on the second example or for a context-sensitive alias analysis on the third example; if it does not distinguish the same variable in two different invocations of foo, it may emit a false positive for a context-sensitive alias analysis on the third example. To reduce such false positives while remaining agnostic to the alias analysis checked, NEONGOBY must provide a very precise DidAlias.

Second, the same observed address of a pointer is not always intended to refer to the same object, which occurs for two reasons. Spatially, pointers may have invalid addresses (e.g., go off bound or are uninitialized). For instance, an off-by-one pointer for marking the end of an array may accidentally have the same address as a pointer to the next object. Off-by-one pointres are allowed by ANSI C specification as long as they are not dereferenced. Therefore, NEONGOBY must handle them to avoid false positives. Temporally, the same piece of memory may be reused for different objects. For instance, two heap memory allocations may return the same address if the first allocation is freed before the second allocation. Thus, NEONGOBY cannot simply claim that two pointers did alias if their addresses are identical; instead, it may need to track whether a pointer is valid and, if so, what object it points to. This problem appears familiar to the problem bounds checkers solve, but it is actually very different: NEONGOBY assumes a test program is largely correct and runs it to detect alias analysis errors, whereas bounds checkers are for preventing buffer overflow attacks. Thus, it is an overkill for NEONGOBY to borrow complex bounds-checking techniques such as tracking base and bounds for each pointer.

A secondary challenge facing NEONGOBY is performance overhead. NEONGOBY is designed to detect errors, so overhead is typically not a big issue. However, NEONGOBY is also designed to detect alias analysis errors that manifest on real-world programs, and large overhead may disturb the executions of these programs [24], such as triggering excessive timeouts. Moreover, different users may have different resource budgets and coverage goals when testing their alias analyses. Since users can best decide what overhead is reasonable, NEONGOBY should provide them the flexibility to make their own tradeoffs between bugs and overhead.

NEONGOBY addresses the false-positive challenge using two ideas. First, NEONGoby considers two pointers alias only when they have the same address simultaneously at a point in an execution. This idea makes DidAlias field-sensitive, flow-sensitive, and context-sensitive ( $\S 2)$.

Second, NEONGOBY bridges the gap between an address and an object by a practical selection of techniques. For instance, NEONGOBY versions memory, so if a piece of memory is reused, the addresses before and after the reuse get different versions; it also pads memory allocation to tolerate off-bound pointers.

With these two ideas, NEONGOBY emits no false positives under two assumptions. First, the test program does not have undefined behavior per ANSI C specification, e.g., use pointers off bound by many bytes. Second, the test program does not call external functions that return reused memory addresses and are not treated as memory allocation functions by NEONGOBY (\$3.1).

NEONGOBY addresses the performance challenge using two ideas. First, NEONGOBY provides two checking modes, enabling a user to select the mode best suited for her alias analysis, test program, and workload. In the offline mode, NEONGOBY logs pointer definitions to disk when running a test program, and checks the log after the test run finishes. Since checking does not slow down the test run, NEONGOBY affords to check more thoroughly: it checks alias queries on pointers potentially in different functions, or interprocedural queries. However, the logging overhead in the offline mode may be high, so NEONGOBY offers another mode to reduce overhead. In the online mode, NEONGOBY checks alias queries on pointers only in the same function, or intraprocedural queries ${ }^{3}$, with efficient inlined assertions, but it may miss some bugs the offline mode catches.

Second, NEONGOBY further reduces performance overhead without losing bugs using an optimization we call delta checking. This optimization assumes a correct baseline alias analysis, such as LLVM's basicaa, often simple enough to have few bugs. NEONGOBY then checks only the pointer pairs that may alias according to the baseline but not the checked alias analysis. By reducing the pointer pairs to check, NEONGOBY reduces overhead.

We implemented NEONGOBY within the LLVM compiler and checked three popular LLVM alias analyses, including (1) the aforementioned basicaa, LLVM's default alias analysis; (2) the aforementioned anders-aa, later used as the basis for two alias analyses [23, 26]; (3) and ds-aa, a context-sensitive, field-sensitive algorithm with full heap cloning [22], later used by [9, 13, 11]. To

\footnotetext{
${ }^{3}$ Intraprocedural queries can still be answered by interprocedural analyses.
} 


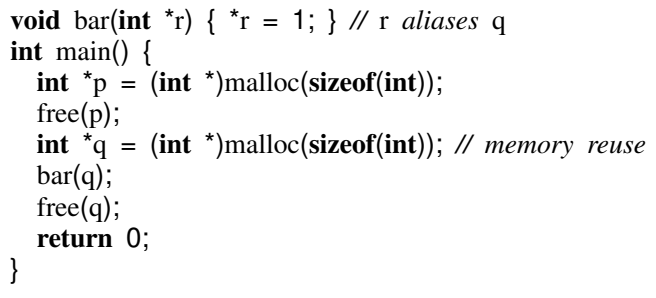

Figure 2: Example test program.

check these analyses, we selected two real-world programs MySQL and Apache and the workloads their developers use. NEONGOBY found 29 bugs in anders-aa and ds-aa, including 24 previously unknown bugs, with zero false positives and reasonable overhead. We have reported eight bugs to ds-aa developers, four of which have been patched $[2,3,4,5]$.

This paper makes four main contributions: (1) our formulation of an approach that dynamically checks general alias analysis with the invariant DidAlias (p, q) $\rightarrow$ MayAlias $(p, q)$; (2) NEONGoBy, a long overdue system toward improving advanced alias analyses into production quality and widening their adoption; (3) a practical selection of techniques to reduce false positives and overhead; and (4) our evaluation results, including real bugs found in two LLVM alias analyses and our proposed patches. Our key inspiration is our anecdotal struggles with some existing alias analyses in our research, so we hope that alias analysis builders can start applying NEONGOBY to improve their alias analyses into productionquality analyses today. As such, we have released it open-source at https://github.com/wujingyue/neongoby, along with our error detection results and proposed patches.

\section{AN EXAMPLE AND OVERVIEW}

Figure 2 shows an example test program. It has three pointers: $p$ and $q$ in function main, and $r$ in bar. Among these pointers, only $q$ and $r$ alias. Suppose buggyaa, a buggy alias analysis misses this only alias pair and reports no alias for all pointers.

To check buggyaa with this test program using the offline mode of NEONGOBY, a user first compiles the code into example.bc in LLVM's intermediate representation (IR), and runs the following three commands:

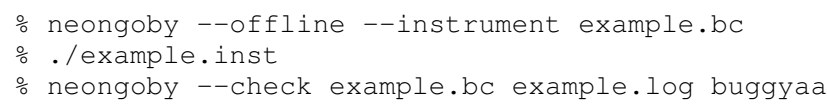

The first command instruments the program for checking: (1) it transforms the program to avoid false positives caused by memory reuse, off-bound pointers, and undefined values and (2) it inserts a logging operation at each pointer definition, memory allocation, function entry and exit to log information for offline checking. The second command runs the instrumented program example.inst to generate a $\log$ of pointer definitions, memory allocations, and function calls and returns. The third command checks this log against buggyaa for errors. It first computes DidAlias for all three pairs of pointers, including pointers not in the same function. It excludes pair $p, q$ and pair $p, r$ from DidAlias even if the two malloc() calls return the same address because the versions of the address are different. It includes pair $q, r$ in DidAlias because $q$ and $r$ share the same address and version. NEONGOBY then checks DidAlias against buggyaa, emitting an error report because MayAlias $(q, r)$ returns false. To diagnose this error, the user can run NEONGOBY to dump log records or slice the log for records explaining why $q$ and $r$ did alias.
To check buggyaa with this test program using the online mode of NEONGOBY, a user runs the following commands:

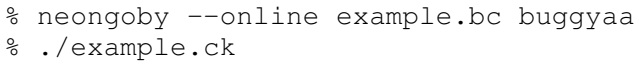

The first command iterates through each function in example.bc, queries buggyaa on each pair of pointers in the function, and, if MayAlias on the two pointers returns false, embeds an assertion that the two pointers never alias at runtime. In this example, NEONGOBY embeds an assertion "assert ( $\mathrm{p} !=\mathrm{q}|| \mathrm{p}==\mathrm{NULL})$ " after the second malloc(). The online mode prevents the two memory allocations from returning the same address using a simple trick of deferring memory deallocation. The second command runs the instrumented program example.ck to check whether this assertion may be triggered, which never happens.

Each mode of NEONGOBY has pros and cons. The offline mode checks more thoroughly, whereas the online mode checks only intraprocedural queries, missing the bug in buggyaa. The offline mode can reuse one log to check multiple alias analyses, amortizing the cost of running the tests, whereas the online mode can check only one alias analysis at a time. However, the offline mode has to log information to disk because the log may grow larger than the RAM for some real-world programs and workloads, and on-disk logging can be costly. In contrast, the inlined assertions the online mode embeds are much faster to check. By providing two modes of operations, NEONGOBY enables a user to select the mode that suits her purpose.

As mentioned in $\S 1$, NEONGOBY makes DidAlias very precise by flagging aliases only when the pointers have the same addresses simultaneously at a point in an execution.

Field sensitivity. DidAlias is field-sensitive, because NEONGOBY reports two pointers alias only when their addresses are identical. For instance, in example 1 in Figure 1, NEONGOBY does not report $p$ aliases $q$ even if they are only one byte apart.

Flow sensitivity. DidAlias is flow-sensitive, because NEONGoBY's offline and online algorithm both consider two pointers alias only when they have the same address simultaneously at a point in an execution. For instance, in the second example in Figure 1, NEONGOBY does not report $\mathrm{p}$ and $\mathrm{q}$ alias even if $\mathrm{p}$ points to ADDR [0] in the first iteration.

Context sensitivity. DidAlias in NEONGOBY's offline detection algorithm is context-sensitive, because the algorithm uses the function calls and returns recorded in the log to distinguish definitions of a pointer in different function invocations, and reports two pointers in the same function alias only when their addresses are identical in the same invocation of that function. For instance, in the third example in Figure 1, although $q$ in the first invocation and $p$ in the second invocation point to the same address, NEONGOBY does not report $\mathrm{p}$ and $\mathrm{q}$ alias because these two pointer definitions belong to different invocations. On the other hand, NEONGOBY's online mode focuses on checking intraprocedural queries, and therefore gains context-sensitivity automatically.

\section{OFFLINE MODE}

This section describes how NEONGOBY operates in the offline mode. Figure 3 shows the offline mode architecture. It has three components: the instrumenter, logger, and offline detector. Given a program in LLVM's intermediate representation (IR), a more lowlevel representation of the program than source code, the instrumenter transforms the program to avoid false positives and inserts logging operations to collect runtime information. When a user 


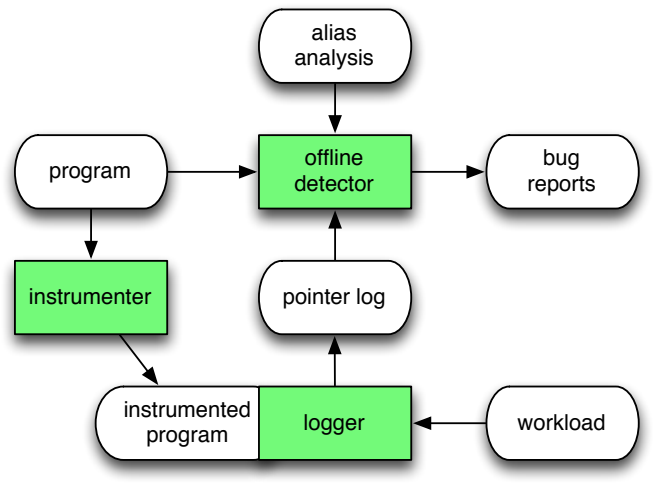

Figure 3: Architecture of the offline mode.

runs the instrumented program, the logger records pointer definitions, memory allocations, function entries and exits to disk. Since the logger runs within a test program, we explicitly designed it to be simple and stateless, reducing runtime overhead and avoiding perturbing the execution of the test program. After the run finishes, the offline detector checks the log against an alias analysis and emits error reports. Since the logger is much simpler than the other components, we focus on describing the instrumenter (\$3.1) and offline detector (\$3.2), and give a brief discussion (\$3.3).

\subsection{Instrumenter}

The instrumenter does five main transformations, the first to ensure that the logger and the offline detector can consistently refer to the pointer variables in a program, the second to collect pointer addresses, and the last three to reduce false positives caused by memory reuse, off-bound pointers, and undefined values. We describe the five transformations below and highlight some of the differences between NEONGOBY and bounds checkers.

Assigning IDs to pointers. The logger and offline detector run in different phases, so they need a consistent way to refer to the pointers in a program. To identify the pointers, the instrumenter traverses the program's control flow graph and assigns to the $n^{t h}$ visited static pointer variable a numeric ID of $n$. To keep IDs consistent, the offline detector uses the same deterministic algorithm (depth-first traversal in our implementation) to assign IDs to pointers. Bounds checkers need not assign IDs to pointers because they cannot defer checking to offline. To check alias analyses more thoroughly, NEONGOBY not only identifies the pointer variables in the source code, but also intermediate pointers in the LLVM IR.

Instrumenting pointer definitions. To catch errors caused by all different types of pointers, NEONGOBY instruments all pointer definitions which assign addresses to pointers. Function pointers and global pointers are particularly crucial: they are widely used in realworld programs such as MySQL and Apache, yet they are often mishandled by alias analyses. Our experiments found 7 bugs caused by mishandled function and global pointers (\$8.1.1).

NEONGOBY logs four types of pointer definitions: pointer assignment $(\mathrm{p}=$ addr where addr can be a pointer arithmetic), pointer load $(\mathrm{p}=*$ addr $)$, function argument passing, and global variable initialization. NEONGOBY instruments all pointer definitions in the LLVM IR, including intermediate results. For instance, LLVM translates $C$ source code $p=0->f$ into two IR instructions

$\circ 1=\% 0+<$ offset of $\mathrm{f}>$

$\div \mathrm{p}=$ load $\% 1$

, and NEONGOBY instruments the definitions of both $\% 1$ and $\%$ p.
NEONGOBY instruments a pointer assignment or load by inserting " $\log _{-}$ptr (ptr, addr)" right after the definition where ptr is p's statically assigned ID, addr is the address assigned, and log_ptr is a logging operation that, when executed, appends the ID and the assigned address to the current on-disk log. NEONGOBY adds calls to log_ptr similarly for function arguments at function entries and for global variables at the entry of the main function. Bounds checkers must also track these pointer definitions to propagate pointer base and bound information.

Instrumenting memory allocations. As discussed in $§ 1$, NEONGOBY cannot use only addresses to compute DidAlias because the same address may point to different objects if memory is reused. To enable the offline detector to handle memory reuse, NEONGOBY instruments all memory allocations, including the allocations of heap, stack, and global variables. (Although global memory cannot be reused, NEONGOBY also instruments it to handle all memory allocations uniformly.) For each allocation, it inserts log_alloc(addr, size) to record the allocation address and size. For stack variables, NEONGOBY inserts log_alloc at the function entry. For global variables, NEONGOBY inserts log_alloc at the entry of main. For heap variables, NEONGOBY inserts log_alloc after a call to one of the following functions:

1. C memory allocation functions: malloc, calloc, valloc, realloc, and memalign;

2. C++ new (mangled names_Znwj,_Znwm,_Znaj, and _Znam); 3. Other library functions: strdup, _-_strdup, and getline.

Users can easily add more heap allocation functions. (One tricky point is that a memory allocation such as " $p=\operatorname{malloc}(\ldots)$ " is also a pointer definition, and NEONGOBY must insert log_alloc before log_ptr for the offline detector to correctly handle memory reuse (\$3.2). Another point is that LLVM shares memory between two constant strings for space if one is the suffix of the other; to avoid these false aliases, NEONGOBY disables this optimization.)

To reduce logging overhead, NEONGOBY does not instrument memory deallocations, such as free() calls, and relies on the offline detector to lazily discover when memory is freed. Thus, a corner case such as "free (q); $p=q$;" may cause NEONGOBY to emit false positives on a flow-sensitive alias analysis that understands free: although $\mathrm{p}$ and $\mathrm{q}$ have the same address, they technically point to nothing. However, use-after-free behavior is undefined per ANSI C standard. Therefore, as long as the test program is correct, NEONGOBY emits no false positives of this type. Bounds checkers in contrast must handle memory deallocations if they want to catch use-after-free errors.

Instrumenting function entries and returns. NEONGOBY need distinguish the same variable in different invocations of a function to reduce false positives on a context-sensitive alias analysis. To track function invocations, for each function, NEONGOBY inserts log_entry (func) at the entry, and log_ret (func) before each return, where func is the ID of the function.

Handling off-bound pointers. Pointers may be assigned offbound addresses that accidentally alias other pointers, causing false positives. Fortunately, most programs use off-bound pointers only to mark the ends of arrays, and these pointers are off by only one byte. This type of off-bound pointer is also the only type allowed by the ANSI C standard. To eliminate false positives caused by off-byone pointers, NEONGOBY transforms a program to add one extra byte for each memory allocation, a technique borrowed from [21]. NEONGOBY currently does not handle other off-bound pointers because they occur very rarely, and we experienced no false positives caused by these pointers in our experiments (\$8.1.2). Bounds checkers in contrast may have to handle these pointers because their 
false positives are fatal and abort executions.

Handling undefined values. Variables may be uninitialized and have undefined pointer values that accidentally look like addresses of other pointers. These values may further propagate through assignments, such as assignments of a struct with an uninitialized pointer field to another struct, causing NEONGOBY to log bogus addresses and emit false positives. NEONGOBY handles undefined pointer values by setting them to NULL because NULL aliases nothing. Resetting undefined pointer values eliminates false positives caused by undefined values as long as the test program is correct, because ANSI C specification disallows using an uninitialized variables, e.g., pointer arithmetics or wild void* and int casts.

\subsection{Offline Detector}

Given a $\log$ of pointer definitions and memory allocations, NEONGoBY's offline detector finds alias analysis errors in two steps: it scans the log to compute the DidAlias results, and then checks DidAlias against an alias analysis and emits error reports.

From a high level, NEONGOBY computes DidAlias results as follows. To distinguish a pointer in different function invocations, NEONGOBY assigns a unique context number to each invocation, and maintains a call stack $C S$ of context numbers. It also maintains two maps: a (conceptual) map $V$ from an address to a version number for handling memory reuse, and a map $P$ from a pointer's unique ID and its context number to an address and version for tracking where pointers point to. We use definition to refer to a pointer-context pair, and location to refer to an address-version pair. Given a log, NEONGOBY scans the records sequentially from the beginning. Upon a memory allocation record, NEONGOBY updates $V$ to assign a new version number for the addresses within the allocated range, so the same addresses get different versions before and after this allocation. Upon a function entry record, NEONGOBY generates a new context number for this invocation, and pushes it to the call stack. Correspondingly, upon a function return record, NEONGOBY pops the call stack so that the caller's context number will be on top. Upon a pointer definition record $l$ with pointer $p t r$ and address $a d d r$, NEONGOBY searches $V$ for $l . a d d r$ 's current version and updates $P$ to make $\langle l . p t r, \operatorname{top}(C S)\rangle$ point to location $\langle l . a d d r, V[l . a d d r]\rangle$. It then searches $P$ for pointer definitions that point to the same location; for each such pointer definition $d$, it adds $\langle l . p t r, d . p t r\rangle$ and $\langle d . p t r, l . p t r\rangle$ to DidAlias unless $d . p t r$ and $l . p t r$ are in the same function and their contexts are different. (Note that $\langle l . p t r, l . p t r\rangle$ is in DidAlias because a pointer aliases itself.)

As discussed in $\S 1$, NEONGOBY must be very precise when computing DidAlias to avoid false positives. The algorithm described above is field-sensitive because it considers that two pointers did alias only when their locations are identical, which requires their addresses to be identical. It is context-sensitive because each invocation gets a unique context number, and two pointers in the same function did alias only when they point to the same location in the same context. It is flow-sensitive because (1) the SSA form of LLVM IR guarantees each pointer is statically defined only once, which provides some flow-sensitivity already, and (2) map $P$ maintains only the latest location of a pointer definition.

Once NeONGoBy computes the DidAlias results, it checks an alias analysis as follows. It iterates through each pointer pair in DidAlias, and checks that the pair is also in MayAlias. It emits an error report otherwise. Since the DidAlias results do not depend on the alias analysis checked, NEONGOBY can reuse them to check multiple alias analyses, amortizing the cost of logging and computing DidAlias.

Our actual algorithm to detect errors offline, shown in Algo-

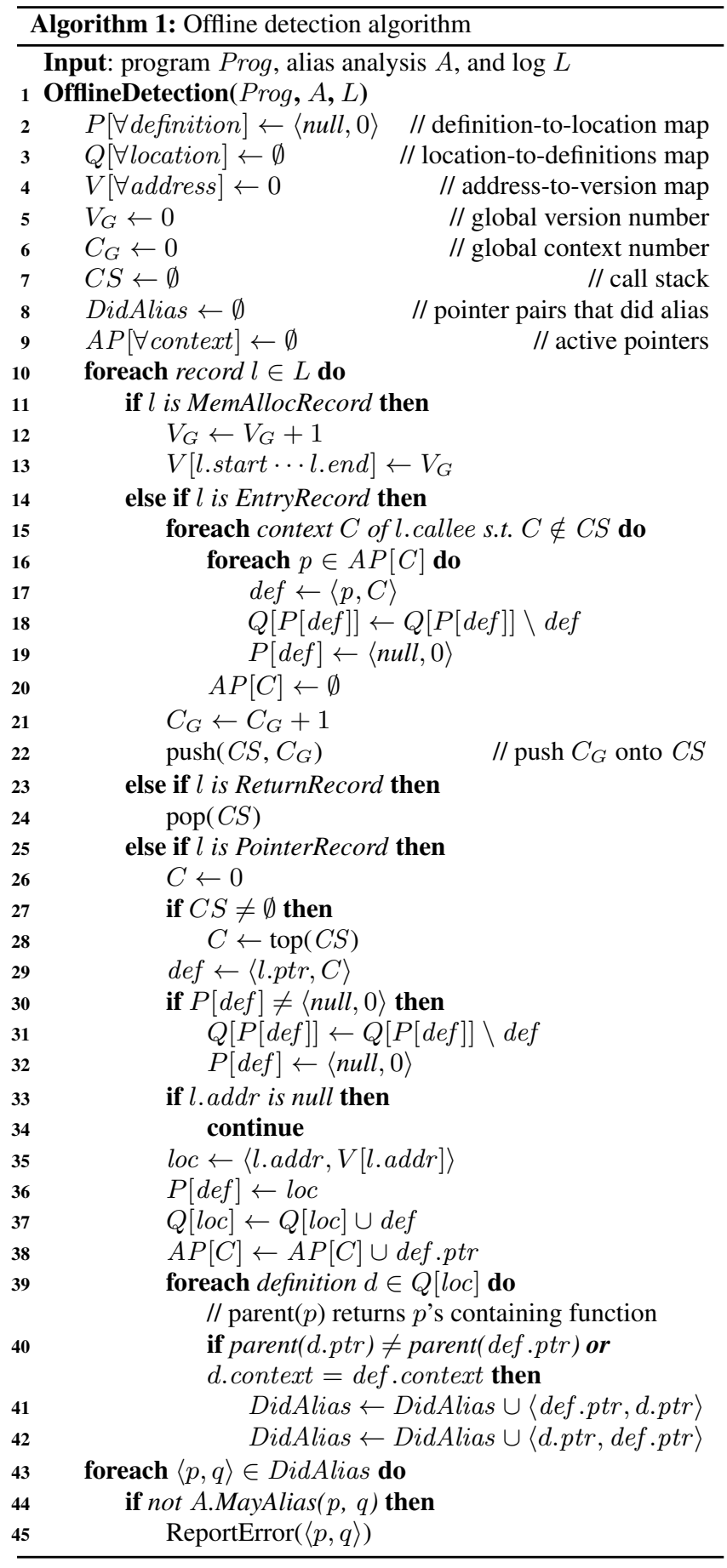

rithm 1, does three optimizations for space and speed. The first optimization implements the address-to-version map $V$ with an interval tree whose key is an address range and value the version number of the entire address range (lines 4, 13, and 35). An interval tree is much more space-efficient than a version number per address. Upon a memory allocation record, NEONGOBY removes $V$ 's existing address ranges that overlap with the allocated range (because these ranges must have been freed), increments a global version number, and inserts the new range and version to $V$. Second, instead of scanning the pointer map $P$ for pointers that point to the same location, NEONGOBY maintains a reverse 


\begin{tabular}{lll} 
& Bounds & NEONGOBY \\
\hline online only & Yes & No \\
use alias analysis & Maybe & No \\
pointer definition & Yes & Yes \\
pointer metadata & Yes & No \\
pointer dereference & Yes & No \\
allocation & Yes & Yes \\
deallocation & Yes & No \\
off-bound-pointer & Yes & Only off-by-one \\
undefined value & Yes & Only pointer
\end{tabular}

Table 1: Different techniques in bounds checkers and NEONGOBY.

map $Q$ from a location back to pointers (lines 3, 18, 31, 37, and 39). Third, when entering a function, NEONGOBY removes from $P$ all outdated pointer definitions in this function (lines 15-20). NeONGoBY considers a pointer definition outdated if its context number is no longer on the call stack. Without removing outdated definitions, $|P|$ could be very large because a pointer can be defined in many function invocations. To efficiently implement this optimization, we use another mapping $A P$ to maintain all active (i.e., non-outdated) pointer definitions indexed by context numbers. With these optimizations, the space complexity of our algorithm is $O(|P|+|M|+\mid$ DidAlias $\mid)$, and the time complexity is $O(|L|(\log |M|+\log |P|+N \log \mid$ DidAlias $\mid))$, where $|M|$ is the number of memory allocations in the $\log$, and $N$ is the maximum size of $Q$ [location]. In our experiments, $N$ never exceeds $400,|M|$ is typically $1 \%$ of $|L|$, and the size of DidAlias is less than $10^{6}$.

\subsection{Discussion}

Some of the problems NeONGoBy addresses, such as tracking pointer definitions and handling memory reuse, off-bound pointers, and undefined values, overlap with what bounds checkers must handle. However, NEONGOBY has very different assumptions and goals than bounds checkers: it assumes a test program is largely correct, and uses the program to detect errors in alias analyses, whereas bounds checkers prevent buffer overflow attacks to a program. False negatives in bounds checkers may lead to exploits, and false positives wrongly abort executions. In contrast, the effects of NEONGoBY's false positives and negatives are much less serious. Because of these differences, it is an overkill for NEONGOBY to borrow complex bounds-checking techniques.

Table 1 summarizes the different techniques in typical bounds checkers and NEONGOBY. Bounds checkers must check buffer overflows online to stop exploits, whereas NEONGOBY can defer costly detection completely offline. Bounds checkers may assume a correct alias analysis (and other static analyses) and use them to remove unnecessary checks, whereas NEONGOBY is intended to detect errors in alias analyses. Bounds checkers need maintain pointer base and bound information with fat pointers, maps, or trees [21], which break backward compatibility or have high overhead. In contrast, NEONGOBY maintains no pointer metadata. Bounds checkers check pointer dereferences and track memory deallocations to catch bugs, whereas NEONGOBY does neither. Bounds checkers may need to accurately handle pointers off by more than one bytes and undefined integer values to avoid wrongly aborting executions, whereas NEONGOBY ignores these cases.

\section{ONLINE MODE}

NEONGOBY's offline mode checks interprocedural alias queries to find more bugs, but its logging may be costly. Thus, NEONGOBY provides an online mode to reduce performance overhead. This section describes how NEONGOBY operates in the online mode.

The online mode focuses on checking intraprocedural queries because they are often considered more crucial than interproce-

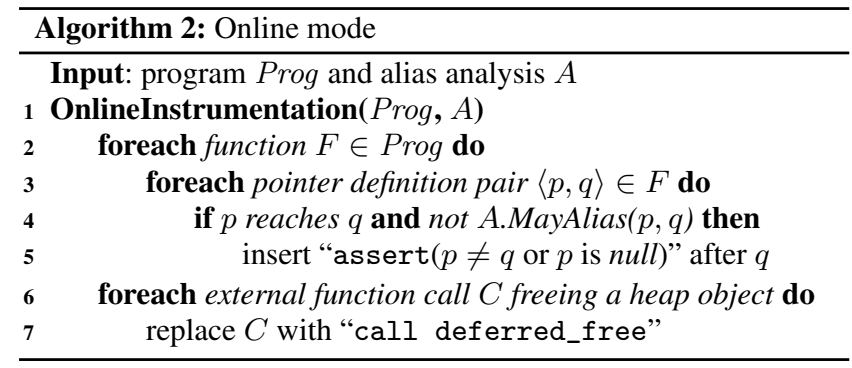

dural queries. For instance, compiler optimizations tend to issue mostly intraprocedural queries. To check intraprocedural queries, NEONGOBY embeds the alias analysis checks as regular program assertions into a test program. NEONGOBY reports an alias analysis bug if one of the assertions fails when a user runs the test program. These assertions are much cheaper than costly on-disk logging at runtime, as shown in our experiments ( $\$ 8.3)$.

Algorithm 2 shows the algorithm to embed the checks. It iterates through each pair of point definitions $p$ and $q$ of a function (line 3 ), and inserts an assertion "assert ( $p$ !=q || $p==$ NULL)" (line 5) if MayAlias (p,q) returns false (line 4). One issue is that the inserted assertion requires that both $p$ and $q$ are defined. NEONGOBY solves this issue with a standard control flow reachability analysis (line 4), and inserts the assertion only if p's definition reaches q. (If pointer $\mathrm{p}$ is undefined along some incoming edges to q's basic block, NEONGOBY creates a new $\phi$-instruction using LLVM's SSA transformation, not shown in Algorithm 2.)

Similar to the offline mode, DidAlias computed in the online mode is very precise. It is field-sensitive, because an assertion fails only when $\mathrm{p}=\mathrm{q}$; it is flow-sensitive, because an assertion uses the latest addresses of the pointers; it is context-sensitive, because it focuses on intraprocedural queries.

To avoid false positives caused by memory reuse, off-bound pointers, and undefined values, the online mode borrows the techniques from the offline mode, with one refinement: it no longer versions memory. The insight is that NEONGOBY checks only intraprocedural queries in the online mode, so it need handle only heap memory reuse, which can be handled in a much simpler way. Specifically, it defers heap memory deallocations so the allocations almost always return different addresses. To do so, it replaces functions that free heap memory, including C's free and C++'s delete (mangled names_ZdlPv and _ZdaPv), with a function that queues the free request without actually freeing memory. When the queue is full, NEONGoby processes half of the queued requests, ensuring that heap memory reuse occurs after at least $n / 2$ free operations where $n$ is the queue capacity. By default, $n$ is $20 \mathrm{~K}$, large enough that no false positives of this type occurred in our experiments.

One additional advantage of the online mode is that the embedded assertions explicitly inform us what to check, enabling NEONGOBY to leverage symbolic execution tools such as KLEE and WOODPECKER [12] to generate inputs that cause the assertions to fail. We leave this for future work.

If a function has an extremely large number (denoted $n$ ) of pointers that do not alias each other, the online mode need insert $O\left(n^{2}\right)$ assertions, which may run slower than the $O(n)$ logging operations inserted by the offline mode. To avoid high overhead caused by such pathological cases, NEONGOBY bounds the number of assertions it inserts to $10^{6}$ for each function, and switches to the offline mode for the function otherwise. In our experiments, we did encounter one such case: a yacc-generated function called MYSQLparse in MySQL needs much more than $10^{6}$ assertions, so NEONGOBY always checks this function offline (\$8.2). 


\section{DELTA CHECKING}

NEONGoBy provides an optimization called delta checking to speed up both online and offline modes without losing any error. The insight is that not all pointer pairs are equally hard to handle by an alias analysis, so NEONGOBY can focus on checking the hard-to-handle pairs and skip the easy ones. To compute what pairs are easy, NEONGOBY takes a user-specified baseline alias analysis assumed to be simple enough to be correct. It then skips checking all pointer pairs $p$ and $q$ on which the baseline's MayAlias $(p, q)$ returns false. Intuitively, if an imprecise baseline alias analysis can infer that two pointers do not alias, then most likely they never alias in any execution, so DidAlias would return false and NEONGOBY would not find any error on the pointers.

We envision two ways this optimization can be used. First, a user specifies an alias analysis she trusts, such as basicaa which computes very conservative alias results, then enjoys speedup without losing errors when applying NEONGOBY to check an advanced alias analysis. Second, an alias analysis builder incrementally checks each precision improvement she makes to her alias analysis. For instance, if her alias analysis reports 10 pointer pairs that each do not alias prior to the improvement and 50 pairs after, she can use NEONGOBY to check this difference of 40 pairs each indeed never alias on some test programs and workloads.

To implement delta checking for the offline mode, we simply change line 44 in Algorithm 1 to

if $B \cdot \operatorname{May} A \operatorname{lias}(p, q)$ and not $A \cdot \operatorname{May} \operatorname{Alias}(p, q)$

where $B$ is the baseline alias analysis. To implement delta checking for the online mode, we simply change line 4 in Algorithm 2 to

if $p$ reaches $q$ and $B$.MayAlias $(p, q)$ and not $A \cdot \operatorname{MayAlias}(p, q)$

Our results using basicaa as baseline show delta checking reduces compilation time, offline detection time, and runtime overhead.

\section{IMPLEMENTATION}

We implemented NEONGOBY in LLVM. It works with version 3.0 and above. It consists of 5,403 lines of C++ code, with 909 for the instrumenter, 168 for the logger, 875 for the offline detector, 642 for the online mode, and the remaining 2,809 for common utilities.

In the remaining of this section, we describe three additional techniques within NEONGOBY: the first to further reduce overhead ( $\$ 6.1)$, the second to help users diagnose error reports $(\$ 6.2)$, and the third to support multiprocess or multithreaded programs (\$6.3).

\subsection{Detecting Errors Using Dereferenced Pointers Only}

Dereferenced pointers are presumably more crucial than the ones not dereferenced, so are the alias results on dereferenced pointers. Thus, NEONGOBY provides users an option to detect alias analysis errors using only dereferenced pointers, including the pointers used in load and store instructions and those passed to external functions because NEONGOBY conservatively assumes that these functions dereference their pointer arguments. Although NEONGOBY with this option may lose some alias analysis errors, it enjoys two benefits. First, the error reports are of higher quality because they are on the more crucial pointers. Second, NEONGOBY runs faster when checking fewer pointer pairs in both offline and online modes. We evaluate this bugs v.s. overhead tradeoff in $§ 8.3$.

\subsection{Simplifying Error Diagnosis}

When NEONGOBY reports an error, it emits two pointers that did alias yet are not marked as aliases by the checked alias analysis. To diagnose such a report, it may be time consuming to manually in- spect all records in the log, so NEONGOBY provides a diagnosis tool to slice the log into a small subset of records that explains why two pointers did alias. The core idea is to trace data dependencies of the two pointers back to a common parent pointer from which both pointers are derived. NEONGOBY traces only direct data dependencies on pointers. For instance, given " $p=q+x$ " where $p$ and $q$ are both pointers, NEONGOBY only traces p's dependency on q, not $x$. Similarly, given " $p=* q$," NEONGOBY only traces $p$ 's dependency on the previous instruction that stores to the address of $q$, and ignores p's dependency on q. NEONGOBY stops tracing back when it finds the common parent pointer or it cannot trace the dependencies further due to (for example) external functions whose source is not available to NEONGOBY. To use this tool on an error report, a user needs to (re)run NEONGOBY's logger (\$3) to log more operations than pointer definitions and memory allocations, including store instructions that store pointer values and call and return instructions of functions that return pointers.

\subsection{Supporting Multiprocess and Multi- threaded Programs}

As discussed in $\S 1$, NEONGOBY is explicitly designed to detect alias analysis bugs that manifest on real-world programs such as Apache and MySQL. These programs often use multiple threads and processes for performance and ease of programming, so NEONGOBY must handle threads and processes. It needs to do so only in the offline mode because the online mode checks intraprocedural queries. Specifically, NEONGOBY shares one log over all threads in a process, and protects the log using a mutex. It assigns one $\log$ to each process. When a process forks, NEONGOBY creates a new $\log$ for the child process. NEONGOBY can then check each $\log$ in isolation. The only modification to Algorithm 1 is maintaining a call stack for each thread. NEONGOBY assumes race freedom as most compilers do, and data races in the worst case may cause some false positives. Fortunately, data races occur so rarely that no false positives of this type occurred in our experiments (\$8.1.2).

\section{LIMITATION}

False positives. NEONGOBY assumes that test programs do not have undefined behavior per ANSI $\mathrm{C}$ specification and may emit false positives on buggy test programs. For instance, NEONGOBY may emit false positives on pointers off bound by many bytes $(\$ 3.1)$ which are disallowed by ANSI C specification. Moreover, NEONGOBY works within a compiler, so external functions that return reused addresses and are not treated as memory allocation functions by NEONGOBY ( $\$ 3.1)$ may cause false positives. For instance, if an external function my_realloc frees and reallocates heap memory, and returns the reallocated memory address, NEONGOBY would miss this memory reuse and emit false positives. However, in practice, external functions seldom cause issues for two reasons: external functions not treated by NEONGOBY as memory allocation functions seldom return reused addresses; without function summaries, alias analyses cannot characterize the address-reusing behavior of those functions either.

False negatives. NeONGoby is a dynamic tool, and detects only alias analysis errors that manifest on the executions it checks. Moreover, we explicitly designed NEONGOBY to be general to check many alias analyses with low false positives, but this generality comes at a cost: NEONGOBY cannot easily find bugs that violate a specific precision guarantee intended by an alias analysis. In our future work, we plan to specialize NEONGOBY's checking toward specific precision guarantees by varying the precision of its DidAlias. In addition, although NEONGOBY checks 
that $\operatorname{DidAlias}(p, q) \rightarrow \operatorname{MayAlias}(p, q)$, it cannot dynamically check that if MayAlias $(p, q)$, then there exists an execution s.t. $\operatorname{DidAlias}(p, q)$, for the following reasons: (1) MayAlias may conservatively return true even if the two pointers never alias in any execution; and (2) even if the pointers do alias in some execution, the given program and workload may not trigger this execution.

\section{EVALUATION}

We evaluated NEONGOBY on three popular alias analyses:

1. basicaa: LLVM's default alias analysis, an intraprocedural, flow-insensitive analysis that collapses all address-taken variables. We chose the version of basicaa in LLVM 3.1.

2. ds-aa: a context-sensitive, field-sensitive alias analysis with full heap cloning [22], actively maintained by LLVM developers. ds-aa is used by $[9,13,11]$. We chose revision 160292 from ds-aa's SVN repo [8].

3. anders-aa: an interprocedural Andersen's alias analysis with three constraint optimizations: hash-based value numbering [16], HU [16], and hybrid cycle detection [15]. We ported the version of anders-aa in LLVM 2.6 to LLVM 3.1. ${ }^{4}$

Both anders-aa and ds-aa have much better quality than typical research-grade analyses; ds-aa in particular is used by many researchers, regularly tested, and actively maintained.

Our test programs are MySQL and Apache, two widespread server programs. Our workloads are benchmarks used by the server developers themselves: SysBench [7] for MySQL, which randomly selects, updates, deletes and inserts database records; and ApacheBench [1] for Apache, which repeatedly downloads a webpage. We compiled these programs and benchmarks with Clang 3.1 and -03. Since MySQL and Apache are server programs, we quantified NEONGOBY's overhead on them by measuring throughput.

Our evaluation machine is a $2.80 \mathrm{GHz}$ Intel dual-CPU 12 -core machine with 64 GB memory running 64-bit Linux 3.2.0. We made both SysBench and ApacheBench CPU bound by fitting the database or web contents in memory; we ran both the client and the server on the same machine to avoid masking NEONGOBY's overhead with network delay; we used four threads for the server and client, and split the total eight threads on different cores to avoid CPU contention.

The remainder of this section focuses on three questions:

\$8.1: can NEONGOBY detect many bugs with zero false positive?

\$8.2: what is NEONGOBY's overhead?

§8.3: what are the bugs v.s. overhead tradeoffs with different NEONGoBY techniques?

\subsection{Bug Detection Results}

This subsection shows the bugs ( $\$ 8.1 .1)$ we found using NEONGoBY and how we detected them (\$8.1.2).

\subsubsection{Bugs Found}

NeONGoby found total 29 bugs, 16 in ds-aa and 13 in anders-aa. Of the 29 bugs, 24 are previously unknown, and four

\footnotetext{
${ }^{4}$ anders-aa was maintained up to LLVM 2.6, so we ported it to LLVM 3.1 with a patch that removes 67 lines and adds 115 . This patch is included in our release of NEONGOBY. It does not change anders-aa's functionality; it merely fixes compatibility issues between LLVM 2.6 and 3.1: it replaces debug output dout with dbgs; migrates anders-aa's handling of an allocation instruction because LLVM 3.1 replaces this instruction with other instructions; adds code to handle a new type of constant (ConstantDataSequential); and changes the alias query interface to include sizes. For each bug found in our port, we verified that the bug also exists in the original anders-aa.
}

ds-aa bugs have been fixed by the developers [2]. Table 2 shows all bugs. Of the 29 bugs, nine $(1-5,17-20)$ are logical bugs; two $(21,22)$ do not handle certain LLVM instructions; the remaining eighteen mishandle external functions or global variables because of analysis incompleteness. Although adding summaries for external functions and global variables can alleviate analysis incompleteness, systematically handling unknown external functions and global variables remains challenging. Researchers and developers strive to make their analyses complete to adopt them into production. For instance, one novel feature of ds-aa is completeness tracking technique to support unknown external functions [22]; anders-aa also strives to handle the incompleteness of external functions (see function AddConstraintsForCall [6]).

We pinpointed the root causes of all bugs in Table 2 to the source. Since anders-aa is relatively simple, we fixed all its detected bugs.

Next we elaborate on two most interesting bugs: bug 1 in ds-aa and bug 17 in anders-aa, both cause the points-to graphs to miss edges, and they require tricky fixes.

Bug 1 is caused by an incomplete call graph traversal in ds-aa. ds-aa constructs its point-to graph in three stages: constructing a local point-to graph for each function, a bottom-up analysis to clone each callee's point-to graph into the caller, and a top-down analysis to merge each caller's point-to graph into the callees. The bottom-up stage computes an unsound call graph $G_{b}$, and the topdown stage computes a sound graph $G_{t}$ based on $G_{b}$ by merging nodes and adding missing edges. Suppose the top-down stage merges node $A$ and $B$ of $G_{b}$ into node $C$ of $G_{t}$. When the top-down stage traverses $G_{t}$, it need traverse both $A$ and $B$ within node $C$. However, the code incorrectly traverses only one of them. We reported this bug to ds-aa developers and they have fixed this bug.

Bug 17 is caused by an incomplete depth-first search (DFS) of the constraint graph in anders-aa's implementation of the HU algorithm. anders-aa answers alias queries by collecting and solving load, store, assignment, and address-of constraints. It organizes these constraints in a constraint graph. It runs HU to identify the points-to sets of pointers and unify the pointers with the same points-to sets. To do so, it runs a DFS over all nodes. It keeps a visited flag per node (Node2Visited), and sets the flag to true when it first reaches the node. As an optimization, when visiting a node representing $* \mathrm{p}$, if the points-to set of the node representing $\mathrm{p}$ is already determined to be empty, anders-aa simply sets the points-to set of $*$ p to be empty. The bug lies in anders-aa's logic to determine when the points-to set of $\mathrm{p}$ is already determined: it wrongly believes the set is determined when p's visited flag is true, even though it has not finished exploring p's descendants or even initialized p's points-to set. We fixed this bug by adding a new flag per node to indicate when DFS has finished exploring the node.

How bugs affect precision. As discussed in $§ 1$, alias analysis bugs may cause tools to mistakenly believe that pointers do not alias when they indeed do, invalidating research findings and compromising safety. To illustrate, we measured how bugs affect alias analysis precision using LLVM's AliasAnalysisEvaluator, which statically queries an alias analysis with all intraprocedural pointer pairs and computes statistics of the results. We define precision as the percentage of queries with no-alias results over all queries. Table 3 shows the precision of basicaa, anders-aa, and the anders-aa after we fixed all its detected bugs. Although anders-aa appears more precise than basicaa on both MySQL and Apache, the fixed anders-aa is actually less precise than the supposedly very imprecise basicaa. This results illustrates that buggy alias results can indeed invalidate evaluation numbers. 


\begin{tabular}{|c|c|c|c|}
\hline \# & AA & File & Description \\
\hline 1 & ds-aa & TopDownClosure.cpp:207 & incomplete call graph traversal in the top-down analysis stage \\
\hline 2 & ds-aa & StdLibPass.cpp:703 & matched formal argument $n$ to actual argument $n+1$ \\
\hline 3 & ds-aa & TopDownClosure.cpp:80 & symptom: missed aliases between actual parameters and the return value of an indirect call \\
\hline 4 & ds-aa & Local.cpp:833 & mishandled variable length arguments \\
\hline 5 & ds-aa & Local.cpp:551 & mishandled inttoptr and ptrtoint instructions \\
\hline 6 & ds-aa & StdLibPass.cpp & did not handle errno; pointers returned from errno may alias \\
\hline 7 & ds-aa & StdLibPass.cpp & did not handle getpwuid_r and getpwnam_r, whose argument and return value alias \\
\hline 8 & ds-aa & StdLibPass.cpp & did not handle gmtime_r-like functions whose return value and the 2 nd argument alias \\
\hline 9 & ds-aa & StdLibPass.cpp & did not handle realpath whose value and the 2 nd argument alias \\
\hline 10 & ds-aa & StdLibPass.cpp & did not handle getenv whose return value aliases for the same environmental variable \\
\hline 11 & ds-aa & StdLibPass.cpp & did not handle tzname, an external global variable \\
\hline 12 & ds-aa & StdLibPass.cpp & did not handle getservbyname whose return values may alias \\
\hline 13 & ds-aa & StdLibPass.cpp & $\begin{array}{l}\text { did not handle pthread_getspecific and pthread_setspecific; the value stored via } \\
\text { pthread_setspecific aliases that loaded via pthread_getspecific with the same key }\end{array}$ \\
\hline $14^{*}$ & ds-aa & StdLibPass.cpp & did not handle strtoll; the dereference of the 2 nd argument may alias the 1 st argument \\
\hline $15^{*}$ & ds-aa & StdLibPass.cpp & did not handle the ctype function family; the return value of __ctype_b_loc-like function may alias \\
\hline $16^{*}$ & ds-aa & StdLibPass.cpp & did not handle freopen whose return value may alias stdin, stdout, or stderr \\
\hline 17 & anders-aa & Andersens.cpp:1882 & HUValNum incorrectly marked a pointer as pointing to nothing. \\
\hline 18 & anders-aa & Andersens.cpp:2588 & mishandled indirect call arguments; points-to edge to argument $n$ may be attached to argument $n \pm 1$ \\
\hline 19 & anders-aa & Andersens.cpp:2585 & points-to nodes representing indirect calls are swapped, but argument info is not updated accordingly \\
\hline 20 & anders-aa & Andersens.cpp:764 & queries on a function pointer and a function always return no alias, even though they do alias \\
\hline $21^{*}$ & anders-aa & Andersens.cpp & did not handle inttoptr and ptrtoint instrucitons \\
\hline $22^{*}$ & anders-aa & Andersens.cpp & did not handle extractvalue and insertvalue instructions \\
\hline 23 & anders-aa & Andersens.cpp:924 & incorrect summary for freopen whose return value may alias the 3 rd argument \\
\hline 24 & anders-aa & Andersens.cpp & did not handle __cxa_atexit \\
\hline 25 & anders-aa & Andersens.cpp & mishandled variable length arguments \\
\hline 26 & anders-aa & Andersens.cpp & did not handle pthread_create \\
\hline 27 & anders-aa & Andersens.cpp & did not handle pthread_getspecific and pthread_setspecific \\
\hline 28 & anders-aa & Andersens.cpp & did not handle strcpy, stpcpy and strcat whose return value aliases the 1 st arguments \\
\hline 29 & anders-aa & Andersens.cpp & did not handle getcwd and realpath \\
\hline
\end{tabular}

Table 2: Descriptions of the bugs found. Starred bugs were either already reported by others or mentioned in the comments of the code. File indicates the file (and the line if there is a clear place to add the fix) containing the bug.

\begin{tabular}{llll} 
& basicaa & anders-aa & fixed anders-aa \\
\hline Apache & $10.9 \%$ & $24.3 \%$ & $10.5 \%$ \\
MySQL & $3.7 \%$ & $5.1 \%$ & $2.7 \%$
\end{tabular}

Table 3: Alias analysis precision. Percentages are no-alias ratios.

\subsubsection{Bug Detection Methodology}

To detect as many bugs as possible, we ran NEONGOBY in the most thorough way: the offline mode without any optimization. ( $\$ 8.3$ shows how the number of bugs varies with different modes and optimizations.)

Our results show NEONGOBY emits no false positives on the three alias analyses and the two applications we checked. We verified all NeONGobY's reports are true positives as follows. For anders-aa, NEONGoBy emitted many reports. Fortunately, one bug typically causes thousands of reports, so we verified the reports as follows. We diagnosed one report, produced a patch, reran NEONGOBY on the patched anders-aa to regenerate reports, mark the reports that disappeared as true positives, and repeated. After about 10 iterations, NEONGOBY emitted no more reports. For ds-aa, NEONGOBY emitted a relatively small number of reports, so we manually inspected each report. Some reports are fairly simple to diagnose, such as incorrect external function summaries. For more complex ones, we created small testcases to reproduce the problems or applied our diagnosis tool (\$6.2) to compute a slice of relevant $\log$ records to simplify diagnosis. We confirmed all ds-aa reports as true positives, and pinpointed the root causes in ds-aa's code for about half of the reports. We could not pinpoint the other reports or reproduce them with small testcases, so we conservatively excluded them from our bug count. Thus, the actual number of ds-aa bugs found may be significantly larger than what we report. We released our bug reports together with NEONGOBY.

\begin{tabular}{l|ll|ll} 
& \multicolumn{2}{|c|}{$\mathrm{ds-aa}$} & \multicolumn{2}{c}{ anders-aa } \\
& MySQL & Apache & MySQL & Apache \\
\hline Bug reports & 395 & 162 & 20,551 & 4,945 \\
Bugs & $10^{*}$ & $7^{*}$ & 13 & 9
\end{tabular}

Table 4: Bug reports and bugs. The second row shows the number of bugs found from the reports. The numbers of ds-aa bugs may be significantly larger than those shown in the table (starred) because we did not count a report as a bug if we could not pinpoint its root cause in the source or reproduce it with a simple testcase.

Table 4 shows the results on ds-aa and anders-aa with our test programs and workloads. (We did not include basicaa because NeONGoby emitted no reports on it.) For ds-aa, NeONGoby emitted 395 reports on MySQL and 162 on Apache. For anders-aa, NEONGOBY emitted 20,551 on MySQL and 4,945 on Apache.

The second row of Table 4 shows the number of bugs found. NEONGOBY found at least $10 \mathrm{ds}-$ aa bugs with MySQL and 7 with Apache. Interestingly, these two sets of bugs only overlap by one bug, illustrating NEONGOBY's benefit of using real-world programs with diverse programming constructs as testing programs. NEONGOBY found 13 anders-aa bugs with MySQL and 9 with Apache, and the Apache bugs are a subset of the MySQL ones.

\subsection{Overhead}

To quantify NeONGoby's overhead, we ran it in the most optimized way: the online mode with all optimizations. ( $\$ 8.3$ shows how the overhead varies with different modes and optimizations.) Table 5 shows the results on basicaa, anders-aa, the fixed anders-aa, and ds-aa. The compilation time of Apache for every checked alias analysis is within 50s. The compilation time of MySQL is relatively longer mostly because anders-aa and ds-aa are slower on MySQL. The throughput highly depends on the pre- 


\begin{tabular}{|c|c|c|c|c|c|c|c|c|}
\hline & \multicolumn{4}{|c|}{ MySQL } & \multicolumn{4}{|c|}{ Apache } \\
\hline & basicaa & anders-aa & fixed anders-aa & ds-aa & basicaa & anders-aa & fixed anders-aa & $\mathrm{ds}-\mathrm{aa}$ \\
\hline Compile & 130.65 & 421.59 & 738.43 & 1714.02 & 19.21 & 41.73 & 22.46 & 39.37 \\
\hline $\mathbf{A A}$ & 8.53 & 213.35 & 656.83 & 1493.30 & 0.53 & 3.93 & 7.01 & 1.90 \\
\hline Insert & 65.30 & 89.60 & 47.72 & 113.26 & 10.35 & 18.88 & 10.49 & 19.07 \\
\hline Codegen & 56.82 & 118.64 & 33.88 & 107.46 & 8.33 & 18.92 & 4.96 & 18.40 \\
\hline TPUT & $59.47 \%$ & $33.32 \%$ & $75.16 \%$ & $34.78 \%$ & $68.05 \%$ & $41.58 \%$ & $81.95 \%$ & $45.62 \%$ \\
\hline Detect & 48.02 & 244.08 & 679.47 & 1536.63 & $\mathrm{n} / \mathrm{a}$ & $\mathrm{n} / \mathrm{a}$ & $\mathrm{n} / \mathrm{a}$ & $\mathrm{n} / \mathrm{a}$ \\
\hline
\end{tabular}

Table 5: NeonGoby's overhead. Compile shows the total compilation time including the time to query the checked alias analysis (AA), insert alias checks (Insert), and generate the executable from the transformed bitcode (Codegen). TPUT shows the relative throughput with NEONGoBY over without. Detect shows the offline detection time for function MYSQLparse; NEONGOBY checks it offline because this yacc-generated function has too many pointers ( $\$ 4$ ). All times are in seconds.

\begin{tabular}{l|lll|lll} 
& \multicolumn{3}{|c|}{ offline } & \multicolumn{3}{c}{ online } \\
& base & delta & delta+deref & base & delta & delta+deref \\
\hline Compile & 3.82 & 3.82 & 2.92 & 226.97 & 189.62 & 41.73 \\
TPUT & $4.83 \%$ & $4.83 \%$ & $11.56 \%$ & $15.52 \%$ & $16.17 \%$ & $41.58 \%$ \\
Detect & 1373.6 & 1204.8 & 579.08 & n/a & n/a & n/a \\
Reports & 4945 & 4945 & 2155 & 3861 & 3861 & 2068 \\
Bugs & 9 & 9 & 8 & 8 & 8 & 6
\end{tabular}

Table 6: Bugs and overhead tradeoffs. The base columns represent the baseline of the offline and online modes; delta with delta checking ( $\$ 5)$; delta+deref with both delta checking and using dereferenced pointers only (\$6.1). The row titles match Table 4 and Table 5. To collect all reports in the online mode, we changed the online mode to emit a report upon an error instead of aborting the current execution.

cision of the alias analysis. For instance, the throughput for ds-aa is smaller than that for basicaa, because ds-aa is more precise. Interestingly, the bugs in anders-aa made it appear very "precise", so its throughput is also small. However, after we fixed all its bugs, its throughput almost doubled. NEONGOBY checks function MYSQLparse offline ( $\$ 4)$, so we also measured this time. Since NEONGOBY logged only operations from MYSQLparse, the log was very small, and most of the offline detection time was spent on querying the checked alias analysis.

\subsection{Bugs and Overhead Tradeoffs}

NEONGOBY provides both the offline and online modes and several optimizations to enable users to flexibly trade bugs for low overhead. This subsection evaluates these tradeoffs using anders-aa because we have understood and fixed all its bugs. We chose Apache as the test program. Table 6 shows the results.

Offline v.s. online. Columns base show that the online mode trades compilation time and a few bugs for significantly increased throughput and reduced detection time. With less than 230s complication time, the online mode improves the throughput of Apache by about three times, and eliminates the offline detection time of about 1500 s. It emits $22 \%$ fewer reports, but misses only one bug. A bug often triggers many reports, so NEONGOBY can still catch a bug as long as some of its reports are emitted.

Delta checking. This optimization improves performance for both the offline and online modes without losing bugs ( 5 ). We chose basicaa as the baseline. Columns delta show that delta checking reduces the detection time by $1.89 \%$ in the offline mode; it reduces compilation time by $16.46 \%$ and increases the throughput by $4.19 \%$ in the online mode. The improvements would be even larger if a user incrementally checks her refinements to her alias analysis.

Detecting errors using dereferenced pointers only. This optimization improves the performance of both modes, but may lose bugs ( $\$ 6.1)$. Columns delta+deref show that this optimization reduces the compilation time by $77.99 \%$ for the online mode; it increases the throughput for both offline and online modes by $80.28 \%$ and $157.14 \%$ respectively; it reduces the offline detection time by $45.62 \%$; and it misses 1 out of 9 bugs in the offline mode, and 2 out

of 8 in the online mode.

\section{RELATED WORK}

Previous sections have discussed how NEONGOBY is related to bounds checkers (or general memory safety tools); this section discusses other related work.

Alias analysis. A plethora of work has been devoted to creating faster, more precise alias analyses [30, 22, 23]. This previous work is complimentary to ours because our goal is to effectively detect errors in alias analysis implementations. There have been several studies on alias analyses, though their focus is on precision and overhead, not correctness. Specifically, Mock et al. [25] measures the precision of static pointer analyses by comparing their results with dynamic points-to sets. However, the dynamic points-to sets they computed are not precise enough for error detection, e.g., the points-to sets are not context-sensitive and they did not handle address reusing. LLVM's AliasAnalysisEvaluator collects statistics about an alias analysis, such as how many pointer pairs do not alias and how many may alias. Hind and Pioli [19] implemented six context-insensitive alias analysis algorithms and compared their precision, time and memory consumption on 24 programs up to 30 $\mathrm{K}$ lines of code. Jablin et al. [20] compared the performance of their system using different alias analyses, and found that the combination of research grade alias analyses [17, 22, 23] sometimes performs worse than the production-quality alias analysis in LLVM.

Software error detection. A plethora of work has also been devoted to software error detection or verification (e.g., $[10,31])$. Most of these systems target general programs, whereas NEONGoB Y targets alias analyses. These analyses take programs as inputs, do complex computations, and compute abstract results with difficult-to-specify guarantees. Thus, prior systems are not directly applicable to detect alias analysis errors. Testing and verifying compilers [28] has also been an important topic for programming language researchers, though, to the best of our knowledge, we are not aware of any prior system for effectively detecting alias analysis errors.

\section{CONCLUSION}

We have presented NEONGOBY, a system for effectively finding alias analysis bugs. NEONGOBY dynamically observes pointer addresses and emits errors if the addresses contradict an alias analysis. Our results show that NEONGOBY can effectively detect many bugs in popular alias analyses with zero false positives and reasonable overhead. Our key inspiration of this work is our anecdotal struggles with some existing alias analyses, so we hope that NEONGOBY can help improve advanced alias analyses into productionquality analyses and vastly widen their adoption. As such, we have released it open-source at https://github.com/wujingyue/ neongoby, along with our error detection results and proposed patches. 


\section{REFERENCES}

[1] ab - Apache HTTP server benchmarking tool. http: //httpd.apache.org/docs/2.2/programs/ab.html.

[2] Bug 12744 - Missing Call Edges, LLVM Bugzilla. http://llvm.org/bugs/show_bug.cgi?id=12744.

[3] Bug 12786 - External Function Summaries, LLVM Bugzilla. http://1lvm.org/bugs/show_bug.cgi?id=12786.

[4] Bug 14147 - Handling of Variable Length Arguments, LLVM Bugzilla. http://llvm.org/bugs/show_bug.cgi?id=14147.

[5] Bug 14190 - Handling Function Pointers, LLVM Bugzilla. http: / / Ilvm.org/bugs/show_bug.cgi?id=14190.

[6] LLVM's Implementation of Andersen's Interprocedural Alias Analysis. http:

//llvm.org/svn/llvm-project/llvm/branches/ release_26/lib/Analysis/IPA/Andersens.cpp.

[7] SysBench: a system performance benchmark. http://sysbench. sourceforge. net.

[8] ds-aa's svn repository, revision 160292. http: //llvm.org/svn/llvm-project/poolalloc/trunk.

[9] T. Bergan, O. Anderson, J. Devietti, L. Ceze, and D. Grossman. CoreDet: a compiler and runtime system for deterministic multithreaded execution. In Fifteenth International Conference on Architecture Support for Programming Languages and Operating Systems (ASPLOS '10), pages 53-64, Mar. 2010.

[10] A. Bessey, K. Block, B. Chelf, A. Chou, B. Fulton, S. Hallem, C. Henri-Gros, A. Kamsky, S. McPeak, and D. Engler. A few billion lines of code later: using static analysis to find bugs in the real world. Commun. ACM, 53:66-75, February 2010.

[11] J. Criswell, A. Lenharth, D. Dhurjati, and V. Adve. Secure virtual architecture: a safe execution environment for commodity operating systems. In SOSP '07: Proceedings of twenty-first ACM SIGOPS symposium on Operating systems principles, pages 351-366, 2007.

[12] H. Cui, G. Hu, J. Wu, and J. Yang. Verifying systems rules using rule-directed symbolic execution. In Eighteenth International Conference on Architecture Support for Programming Languages and Operating Systems (ASPLOS '13), 2013.

[13] D. Dhurjati, S. Kowshik, and V. Adve. SAFECode: enforcing alias analysis for weakly typed languages. In Proceedings of the ACM SIGPLAN 2006 Conference on Programming Language Design and Implementation (PLDI'06), pages 144-157, 2006.

[14] I. Dillig, T. Dillig, and A. Aiken. Sound, complete and scalable path-sensitive analysis. In Proceedings of the ACM SIGPLAN 2008 Conference on Programming Language Design and Implementation (PLDI'O8), 2008.

[15] B. Hardekopf and C. Lin. The Ant and the Grasshopper: fast and accurate pointer analysis for millions of lines of code. In Proceedings of the ACM SIGPLAN 2007 Conference on Programming Language Design and Implementation (PLDI '07), pages 290-299, 2007.

[16] B. Hardekopf and C. Lin. Exploiting pointer and location equivalence to optimize pointer analysis. In 14th International Static Analysis Symposium (SAS '07), pages 265-280, 2007.

[17] B. Hardekopf and C. Lin. Semi-sparse flow-sensitive pointer analysis. In Proceedings of the 36nd Annual Symposium on Principles of Programming Languages (POPL '09), pages
226-238, 2009.

[18] N. Hasabnis, A. Misra, and R. Sekar. Light-weight bounds checking. In Proceedings of 10th International Symposium on Code Generation and Optimization (CGO '12), 2012.

[19] M. Hind and A. Pioli. Evaluating the effectiveness of pointer alias analyses. In Science of Computer Programming, pages 31-55, 1999.

[20] T. B. Jablin, J. A. Jablin, P. Prabhu, F. Liu, and D. I. August. Dynamically managed data for CPU-GPU architectures. In Proceedings of 10th International Symposium on Code Generation and Optimization (CGO '12), 2012.

[21] R. W. M. Jones and P. H. J. Kelly. Backwards-compatible bounds checking for arrays and pointers in $\mathrm{C}$ programs. In Proceedings of The Third International Workshop on Automatic Debugging (AADEBUG '97), pages 13-26, 1997.

[22] C. Lattner, A. Lenharth, and V. Adve. Making context-sensitive points-to analysis with heap cloning practical for the real world. In Proceedings of the ACM SIGPLAN 2007 Conference on Programming Language Design and Implementation (PLDI '07), 2007.

[23] O. Lhoták and K.-C. A. Chung. Points-to analysis with efficient strong updates. In Proceedings of the 38nd Annual Symposium on Principles of Programming Languages (POPL '11), pages 3-16, 2011.

[24] D. Marino, M. Musuvathi, and S. Narayanasamy. Literace: Effective sampling for lightweight data-race detection. In Proceedings of the ACM SIGPLAN 2009 Conference on Programming Language Design and Implementation (PLDI '09), June 2009.

[25] M. Mock, M. Das, C. Chambers, and S. J. Eggers. Dynamic points-to sets: a comparison with static analyses and potential applications in program understanding and optimization. In Proceedings of the 2001 ACM SIGPLAN-SIGSOFT workshop on Program analysis for software tools and engineering (PASTE '01), pages 66-72, June 2001.

[26] R. Nasre and R. Govindarajan. Prioritizing constraint evaluation for efficient points-to analysis. In Proceedings of the 9th Annual IEEE/ACM International Symposium on Code Generation and Optimization (CGO'11), pages 267-276, 2011.

[27] D. Prountzos, R. Manevich, K. Pingali, and K. S. McKinley. A shape analysis for optimizing parallel graph programs. In Proceedings of the 38nd Annual Symposium on Principles of Programming Languages (POPL'11), pages 159-172, Jan. 2011.

[28] J. Regehr, Y. Chen, P. Cuoq, E. Eide, C. Ellison, and $\mathrm{X}$. Yang. Test-case reduction for $\mathrm{C}$ compiler bugs. In Proceedings of the ACM SIGPLAN 2012 Conference on Programming Language Design and Implementation (PLDI '12), pages 335-346, 2012.

[29] L. Shang. Pointer analysis in pldi/popl from 1998. http: / / www. cse. unsw. edu. au/ shangl/topconf. htm.

[30] J. Whaley and M. S. Lam. Cloning-based context-sensitive pointer alias analysis using binary decision diagrams. In Proceedings of the ACM SIGPLAN 2004 Conference on Programming Language Design and Implementation (PLDI '04), pages 131-144, June 2004.

[31] J. Yang, C. Sar, and D. Engler. Explode: a lightweight, general system for finding serious storage system errors. In Proceedings of the Seventh Symposium on Operating Systems Design and Implementation (OSDI '06), pages 131-146, Nov. 2006. 